\title{
Polyglutamine toxicity in non-neuronal cells
}

\author{
Jennifer W Bradford ${ }^{1}$, Shihua $\mathrm{Li}^{1}$, Xiao-Jiang $\mathrm{Li}^{1}$ \\ ${ }^{1}$ Department of Human Genetics, Emory University School of Medicine, Atlanta, GA 30322, USA
}

The neurodegenerative polyglutamine diseases are caused by an expansion of unstable polyglutamine repeats in various disease proteins. Although these mutant proteins are expressed ubiquitously in neuronal and non-neuronal cells, they cause selective degeneration of specific neuronal populations. Recently, increasing evidence shows that polyglutamine disease proteins also affect non-neuronal cells. However, it remains unclear how the expression of polyglutamine proteins in non-neuronal cells contributes to the course of the polyglutamine diseases. Here, we discuss recent findings about the expression of mutant polyglutamine proteins in non-neuronal cells and their influence on neurological symptoms. Understanding the contribution of non-neuronal polyglutamine proteins to disease progression will help elucidate disease mechanisms and also help in the development of new treatment options.

Keywords: polyglutamine, Huntington's disease, neurodegeneration, glia, misfolding, aggregation

Cell Research (2010) 20:400-407. doi: 10.1038/cr.2010.32; published online 16 March 2010

\section{Introduction}

There are at least nine inherited neurodegenerative diseases that are caused by an expansion of a polyglutamine (polyQ) tract, including Huntington's disease (HD), spinocerebellar ataxia (SCA) 1-3, 6, 7 and 17, spinobulbar muscular atrophy (SBMA), and dentatorubropallidoluysian atrophy (DRPLA) $[1,2]$. The polyQ expansion results from a CAG triple repeat expansion in unique genes that do not share homology. Despite this, these diseases share many common pathological features. First, symptoms of these diseases usually appear at midlife and progressively worsen until death, some 15-20 years later. A juvenile, or early-onset form, also exists for these polyQ diseases, and is usually associated with more than $60 \mathrm{CAG}$ repeats. Longer repeat sizes are associated with an earlier age of disease onset. Second, these diseases all show aggregates or inclusions formed by mutant proteins in the brain, which are a pathological hallmark of the polyQ diseases. It remains debated as to whether aggregates are toxic or have a protective role, but they do reflect the accumulation of mutant polyQ proteins in cells. Third, expression of these mutant proteins is generally widespread throughout the body, but

Correspondence: Xiao-Jiang Li

Tel: +1-404-727-3290; Fax: +1-404-727-3949

E-mail: xli2@emory.edu selective degeneration is seen in specific brain regions. Although these diseases cause neuronal degeneration, evidence is growing that expression of mutant proteins in non-neuronal cells might also contribute to disease pathology $[3,4]$.

\section{Polyglutamine diseases and selective neuropathology}

\section{Huntington's disease}

HD is the most common polyQ disorder with an incidence of around 70 per million people in people of Western European descent, but its prevalence is lower in the rest of the world. The expanded polyQ domain is located in the N-terminal region of the HD protein, huntingtin (htt). Individuals having more than $36 \mathrm{CAG}$ repeats will develop HD [5, 6], and symptoms usually consist of body weight loss, psychiatric problems, cognitive deficits, and movement disorders (chorea), which are followed by death that occurs 10-20 years after onset. The selective neurodegeneration in HD occurs early in the medium spiny neurons in the striatum. Other brain regions such as the deep layers of the cortex, the hypothalamus, and hippocampus also undergo neurodegeneration, which is widespread in the late stages of HD [7]. Htt is normally a cytoplasmic protein and is essential for cell survival [8]. While the primary function of htt has yet to be elucidated, it probably plays a role in cellular trafficking and also functions as a scaffold protein $[9,10]$. It is evident that only $\mathrm{N}$-terminal mutant $\mathrm{htt}$ is able to form aggregates and 
is more toxic than full-length mutant htt, which has led to extensive studies to identify the proteolytic cleavage sites. These studies have identified that various proteases, including calpains and caspases, can degrade mutant htt to generate $\mathrm{N}$-terminal htt fragments [11-15]. In addition to having the properties to misfold and aggregate, $\mathrm{N}$ terminal mutant htt fragments can also accumulate in the nucleus, while the majority of full-length mutant htt remains in the cytoplasm [16-18]. The nuclear localization of N-terminal mutant htt can lead to abnormal binding of mutant htt to various transcription factors, which will subsequently affect transcriptional expression $[10,19]$.

\section{Spinocerebellar ataxias}

This group of polyQ neurodegenerative disorders includes six (SCA 1, 2, 3, 6, 7, and 17) identified diseases [1], which compose about $50 \%$ of all families affected by SCA [20]. These diseases are all dominantly inherited and characterized by cerebellar degeneration, which results in ataxia, speech difficulties, dementia, and eventually death [1]. SCA1 is caused by a polyQ expansion of $>43$ repeats in the ataxin-1 gene [21]. SCA2 is caused by the ataxin- 2 gene containing $>37$ polyQ repeats [22] SCA3, also called Machado-Joseph disease, is the most common form of the ataxias and occurs when the disease protein ataxin-3 has $>50$ repeats [23]. The SCA6 gene encodes a voltage-dependent calcium channel (CACNA1A), which is a membrane channel protein that contains a polyQ domain that expands to cause SCA6 [20, 24]. SCA7 results from the expansion of the CAG/polyQ repeat ( $>37$ units) in the ataxin-7 gene [25]. One interesting point about SCA7 is that expression of ataxin-7 is high in peripheral tissues, such as heart, skeletal muscle, and pancreas, and lower in the brain. Also, SCA7 is associated with cone-rod dystrophy retinal degeneration [26]. SCA17 occurs when there is an expansion of 42 or more repeats in the polyQ tract of the TATA-box binding protein (TBP), which plays an essential role in transcriptional regulation. Despite the critical role of TBP in cellular function in general, SCA17 also shows selective neurodegeneration associated with cerebellar atrophy, in particular, Purkinje cell death [27].

Spinobulbar muscular atrophy and dentatorubropallidoluysian atrophy

Two other polyQ diseases, SBMA and DRPLA, have also been well characterized. SBMA, also known as Kennedy disease, occurs when there is a polyQ expansion of 36 or more repeats in exon 1 of the androgen receptor (AR) gene $[28,29]$. SBMA is the only X-linked, recessively inherited disease in the polyQ neurodegenerative disease family, and primarily affects males [30]. This disease is characterized by motor neuron loss in the spinal cord and brain stem, and leads to a slowly progressing disease with onset in middle age [31]. Symptoms of SBMA include muscular atrophy and weakness, particularly of the limbs and facial muscles, reduced male fertility, and death that often occurs due to pneumonia [29, 32]. Interestingly, AR expression is lower in the central nervous system (CNS) than in cardiac and skeletal muscle, skin, and prostate [30].

DRPLA is a dominantly inherited neurodegenerative disease that occurs when the atrophin-1 protein has an expanded polyQ tract containing $>48$ repeats $[33,34]$. DRPLA transgenic mice exhibit symptoms including ataxia, chorea, and dementia [35]. Atrophin-1 is a transcriptional regulator and is expressed ubiquitously in different types of cells [34], but mutant atrophin-1 causes neuronal degeneration in select neuronal populations in the cerebellum [35].

\section{Glial dysfunction in polyglutamine diseases}

\section{Huntington's disease}

Glial cells, once only considered as supporting cells, are now recognized as vital components of the CNS and carry on many functions that support neuronal function and survival. Glial cells compose $90 \%$ of cells in the brain and consist of three different types: astrocytes, microglia, and oligodendrocytes [36]. Astrocytes and microglia, in particular, have been implicated in playing a role in polyQ disease pathology.

Evidence that glial cells express mutant htt and display htt aggregates comes from several different groups. In the R6/2 HD mouse model, exon1 htt with 115-150 CAG repeats is expressed in both neurons and glia [37, 38]. In addition, examination of HD human patient brains also reveals the presence of mutant htt in glial cells [37, 39]. HD patients have also been found to have microstructural changes in the white matter of the corpus callosum [40]. However, fewer glia than neurons contain aggregates, and glial aggregates are usually smaller than neuronal ones in HD transgenic and knock-in mice [37, 41]. There are several explanations that would account for fewer glia containing smaller aggregates than neurons. One possibility is that glial cells intrinsically express $h t t$ at a lower level than neuronal cells, though this possibility remains to be verified. Another possibility is that unlike neurons, glial cells are capable of dividing, a process that could deplete the amount of mutant protein in the cell. Our recent studies have found that ubiquitin-proteasome system (UPS) activity is lower in neurons than in glia, and that UPS activity levels decrease with age [42]. The UPS is responsible for clearing misfolded protein, and its lower activity in neuronal cells could result in increased 
accumulation of N-terminal mutant protein, and subsequently, htt aggregation in neurons. Age-related decline in UPS activity in neurons and glial cells could explain age-dependent formation of polyQ aggregates and disease progression. The more abundant accumulation of mutant polyQ proteins in neurons than in glial cells could also in part explain the selective neuronal loss common to these polyQ diseases.

The presence of toxic proteins in glial cells could affect their function. Because astrocytes play important roles in maintaining synaptic plasticity and neuronal communications $[36,43]$, astrocyte dysfunction could be deleterious to surrounding neurons. One of the most important roles of astrocytes is to remove excess synaptic glutamate, which is the major excitatory neurotransmitter in the CNS [43]. GLT-1 and GLAST are the major astrocyte glutamate transporters (human homologs are EAAT-2 and EAAT-1, respectively) that remove extracellular glutamate. Proper glutamate uptake protects the surrounding neurons from excitotoxicity that could occur if excess glutamate remains in the synaptic cleft $[36,43]$. The R6/2 mouse shows a significant decrease in GLT-1 transcript and protein levels in the striatum and cortex at 12 weeks of age, but not at 4 weeks, while a decrease in GLAST levels has not been indicated in HD [44, 45]. Through microdialysis in freely moving R6/2 mice, it was found that the $\mathrm{R} 6 / 2$ mice had a progressive reduction in GLT-1 function [45]. Importantly, the striatum region of HD patients shows decreased GLT-1 mRNA levels and decreased glutamate uptake [46, 47].

A Drosophila model of HD was also created to examine the potential roles of glial dysfunction in HD pathology [48]. In this study, the UAS-GAL4 system was used to express exon1 of human htt with 20 or 93 CAG repeats in dEAAT1-expressing glia, which are the glial subtype that express the sole glutamate transporter in the fly. Although the transgenic flies died, degeneration was not seen in the glial cells. This implies that glial dysfunction due to mutant htt expression and not glial degeneration probably contributed to the phenotypes seen. We have recently generated transgenic mice that express mutant $\mathrm{N}$-terminal htt specifically in astrocytes. These mice show age-dependent neurological symptoms without obvious neurodegeneration [49]. Since medium spiny neurons in the striatum, the neurons that are preferentially killed in HD, are innervated by glutamatergic axons from the neocortex, a decrease in astrocyte glutamate uptake might make them more susceptible than other types of neurons to excitotoxicity, which at least can result in neuronal dysfunction.

\section{SCA7}

The involvement of glia has also been implicated in
SCA7 disease pathology. A mouse model that expresses mutant ataxin-7 in Bergmann glia was developed to study this role [50]. Bergmann glia are a type of astrocytes that are located in the cerebellum and are closely associated with Purkinje neurons. Like other astrocytes, they are responsible for removing extracellular glutamate from the synapse. This mouse model revealed that expression of ataxin-7 in Bergmann glia alone was sufficient to cause Purkinje cell pathology and impair motor coordination, as measured by the rotarod test [50]. Like the HD mouse models studied, this SCA7 model also implies an important role for glial cells in polyQ disease progression.

\section{Reactive glia in poly $Q$ diseases}

During CNS injury or disease, astrocytes undergo a process termed reactive gliosis, which is characterized by up-regulation of glial fibrillar acidic protein (GFAP), cell proliferation, and morphology changes [51]. GFAP is an intermediate filament protein that is expressed in astrocytes and is commonly used as an astrocytic marker [52]. Increases in GFAP are an early indication of reactive gliosis, and neuronal dysfunction can be induced by defective astrocytes, even in the absence of neurodegeneration [53]. Reactive astrocytes occur in the striatum and increase in number with HD disease progression [7, $54,55]$. Reactive gliosis occurs even in HD models that do not express mutant htt in neurons [49], indicating that mutant htt expression in astrocytes alone is sufficient to cause this glial response.

Microglia are glial cells that function as immune cells in the CNS, and like astrocytes, can also become reactive. Microglia normally exist in a quiescent state until they are exposed to an insult or injury, which includes protein aggregation in HD [56]. Following injury, these cells become activated and will transform into phagocytes, which are able to remove dying cells and will release proinflammatory cytokines, proteases and oxygen radicals to combat the insult $[54,57]$. It has been previously shown that microglia activation often precedes neuronal degeneration in many neurodegenerative diseases such as Alzheimer's disease and Parkinson's disease [58]. HD patient brain regions, and in particular, the striatum and cortex, have been shown to contain activated microglia, with activation increasing as the severity of disease increases [54]. Activation of astrocytes and microglia in polyQ diseases reiterates that non-neuronal cells can be impacted by the presence of mutant polyQ proteins.

\section{Dysfunction of peripheral tissues in polyQ diseases}

Recently, there has been an increase in data showing 
that polyQ diseases affect not only cells in the CNS but also peripheral tissues. Although the majority of work has been performed using the R6/2 mouse model to show the presence of htt aggregates in peripheral tissues, a new study demonstrates that an HD knock-in mouse, which expresses full-length mutant htt (HdhQ150), also has mutant htt aggregates in various peripheral tissues [59]. As additional mouse models and human patient samples are studied, more light will be shed on how polyQ diseases affect such a wide range of cells, which will help the development of treatments.

\section{Heart}

Approximately $24 \%$ of HD patients die from cardiac failure, which is the second most common cause of death, following pneumonia [60]. The R6/2 HD mouse model has recently been studied for signs of cardiac problems due to mutant htt expression [61]. By electron microscopy, significant elevation in htt aggregation was found in the nucleus and mitochondria in cardiac myocytes of these HD mice. Other important findings include cardiac structural remodeling, reduced cardiac output by $50 \%$ by 12 weeks of age, decreased heart weight, and altered mitochondrial ultrastructure in the R6/2 mouse compared to littermate controls [61]. It has also been found that cardiomyocytes isolated from human heart failure patients contain pre-amyloid oligomers, which are soluble intermediates of protein aggregates [62]. This finding led researchers to question whether the presence of soluble polyQ oligomers expressed in the heart could produce toxic effects. To help answer this question, a transgenic mouse model was developed to express expanded polyQ preamyloid oligomers specifically in cardiomyocytes under the control of the $\alpha$-myosin heavy chain promoter [63]. Investigation of this mouse model revealed that expression of expanded polyQ preamyloid oligomers caused formation of protein aggregates in cardiomyocytes, which led to cardiac dysfunction and dilation, as well as reduced lifespan [63]. These studies show that mutant polyQ proteins expressed in cardiomyocytes, either as aggregates or as soluble oligomers, can contribute to cardiomyocyte death and heart failure. This finding helps explain the large percentage of HD patient deaths due to cardiac failure and gives more evidence for the importance of non-neuronal cells in polyQ disease pathology.

\section{Pancreas}

It has been recognized for some time now that the risk of developing diabetes mellitus is significantly higher in HD patients than in the general population $[64,65]$, as well as having decreased glucose tolerance [66]. The $\beta$ cells of the islets of Langerhans of the pancreas are responsible for normal production of insulin, which is critical for maintaining blood glucose homeostasis [67]. Like other peripheral tissues, $\beta$ cells also express wild type and mutant htt. Thus, several HD mouse models, including the R6/1, R6/2, and KI models, have been studied to see whether the mice display diabetes phenotypes.

The R6/2 model that ubiquitously expresses exon 1 mutant htt displayed impaired glucose tolerance by 8 weeks of age and developed diabetes mellitus by 12 weeks with fasting hyperglycemia and strikingly abnormal glucose tolerance [67]. Insulin mRNA levels were found to decrease in the pancreas of the R6/2 mice as disease progressed, and this decrease is evident prior to the development of diabetes mellitus. Decreased insulin mRNA levels might be due to altered expression of insulin gene expression regulators (PDX-1 and p300) by mutant htt [67]. Another group also studied the R6/2 model of HD and found that as the mice aged, $\beta$ cell mass did not increase, due to reduced replication, and insulin secretion was deficient when compared to wildtype mice [68]. The investigators reasoned that low insulin secretion in the R6/2 mouse was probably due to reduced exocytosis that resulted from a decreased number of secretory vesicles in the R6/2 $\beta$ cells [68]. The fulllength mutant htt knock-in mouse model, $H d h \mathrm{Q} 150$, was found to contain htt aggregates in $\beta$ cells of the pancreas, but overall, pancreatic pathology seemed less severe than what was observed in the R6/2 model [59].

The R6/1 mouse, which is the same as the R6/2 mouse but with a shorter CAG repeat, has also been examined for diabetes phenotypes. It was found that while this model does not develop diabetes, the R6/1 mice do show reduced $\beta$ cell mass and abnormal insulin release [69]. The N171-82Q mouse model [35], which expresses Nterminal mutant htt with $82 \mathrm{CAG}$ repeats in neurons, also has elevated plasma glucose levels and abnormal glucose tolerance $[3,70]$. All of these results suggest that mutant $h t t$ has a negative impact on normal pancreas function.

\section{Adipose}

Body weight loss is a prominent symptom in HD patients and mouse models and can become very severe in later disease stages. Body weight loss can still occur under very high caloric intake [71], and HD patients who have a higher body mass index will usually have slower disease progression [72]. One common explanation of the dramatic wasting seen in HD patients is that mutant htt may cause metabolic defects that either contribute to or result from neurodegeneration [73]. In N171-82Q HD mice, neurodegeneration also occurs in the hypothalamus, a region that influences food intake 
and energy expenditure [74]. To help explain such severe wasting, several groups have examined whether there are any changes in the adipose tissue of HD mouse models. Adipose tissue is composed of adipocytes (fat cells) that help regulate glucose homeostasis by producing adipokine hormones such as leptin and adiponectin [71]. The leptin hormone functions in the hypothalamus to regulate food intake and energy balance [75], and adiponectin functions in the skeletal muscle to promote proper glucose uptake [71]. The R6/2 model has a tendency to gain weight prior to the onset of symptoms, which may be attributable to the inability of adipocytes to properly break down fat [73]. Even with an increase in weight prior to the onset of phenotypes, the R6/2 mouse still displays wasting in later disease progression. A study using the Huntington Study Group data sample has shown that HD patients with minimal impairment (early in disease progression) have a lower body mass index than sex- and age-matched control groups [76]. This finding negates a previous hypothesis that weight loss results from increased energy expenditure due to severe body movements, because weight loss occurs prior to the onset of severe chorea. Thus, weight loss seen in HD might be due to metabolic reasons. It has recently been found that the R6/2 HD mouse model, which has a short lifespan, and the CAG140 knock-in model, which has a longer lifespan, both have dysfunctional adipose tissue, even at early ages [71]. In both of these models, leptin and adiponectin expression levels and circulating levels were decreased when compared to wild-type mice in later disease stages. The expression of several genes involved in adipocyte maturation was found to be significantly decreased in the R6/2 mouse in later disease stages [71], which could lead to adipocyte dysfunction and severe weight loss. Recently, a group transplanted adipose-derived stem cells into the R6/2 mouse model and quinolinic acid-induced HD rat model. They found that the transplant slowed striatal degeneration, improved behavioral effects such as clasping, male body weight loss, rotarod performance, and increased survival in the R6/2 mouse [77]. Interestingly, adipose-derived stem cell transplantation also increased levels of PPAR gamma coactivator 1alpha (PGC-1 $\alpha$ ), which plays several roles in energy metabolism, and is known to be decreased in HD patients [77]. Targeting treatment to dysfunctioning adipocytes may lead to improved quality of life and survival for HD patients.

\section{Muscle}

Advanced HD is associated with progressive skeletal muscle atrophy [19], and htt aggregates have been found in muscle nuclei of the R6/2 mouse model $[78,79]$, and in a full-length htt knock-in mouse model of HD with 150 glutamines [59]. In humans [80] and HD mouse models [81], motor deficits begin before significant body weight loss occurs, which implies that loss of motor ability is not primarily attributable to weight loss. Even though the R6/2 mouse lives between 12-15 weeks, an extensive study of the motor coordination and strength of the R6/2 mouse showed that muscular phenotypes presented as early as 5-6 weeks [81]. Examination of the muscle of the R6/2 mouse revealed that motor neurons and muscle fibers are affected by the presence of mutant htt. Severe atrophy of the muscle fibers was seen in late stage animals, although cell death was not found [79].

Microarray analysis of R6/2 and human HD patient muscle biopsies revealed that some common muscle genes had altered expression patterns [80, 82]. For example, the lactate dehydrogenase $A$ gene, which is the major $\mathrm{LDH}$ isoform in skeletal muscle, was found to have decreased expression in both mouse and HD patients [80]. In addition, the function of PGC- $1 \alpha$ in HD mouse muscles is impaired, and was found to be decreased in HD patient muscles as well $[83,84]$. The altered expression profiles seen in HD patient and mouse models indicate that mutant htt has a deleterious effect on muscle cells, which could help explain the severe muscle atrophy seen in HD.

\section{Drug studies aimed at targeting the peripheral ef- fects of polyQ diseases}

HD transgenic mouse models are very useful for finding effective therapeutics [85], and there have been studies that attempt to treat some pathological changes seen in HD peripheral tissues. Creatine is administered as a drug to help with metabolic problems and also has neuroprotective effects [70]. Creatine was found to significantly extend survival, improve motor performance, delay body and brain weight loss, and slow the onset of diabetes and accumulation of aggregates in the brain and pancreas of the R6/2 [86] and N171-82Q [70] HD mouse models. Another drug used to treat diabetes in HD mouse models is exendin-4, which is an FDA-approved antidiabetic glucagon-like peptide 1 receptor agonist. Daily treatment of the N171-82Q HD mouse model with exendin-4 resulted in significantly reduced blood glucose levels in the mice [87]. Treatment also improved weight loss and rotarod ability of the mice, which are two parameters that are used to judge phenotype onset. Ceftriaxone and other $\beta$-lactam antibiotics that increase GLT-1 levels have also been used to improve glial function in HD mouse models. Ceftriaxone, in particular, has been found to effectively increase glial GLT-1 levels and to also improve 
behavioral phenotypes in the R6/2 HD mouse model [88]. Drugs aimed at targeting the peripheral effects of polyQ diseases may be easier to administer or may have a more pronounced effect than current drugs aimed at treating neurons.

\section{Closing remarks}

It is evident that neuronal and non-neuronal cells express mutant polyQ proteins, and we are beginning to see the effects of mutant polyQ proteins on non-neuronal cells. An important question is why neurons are selectively killed in these diseases. Neurons are probably more sensitive to mutant proteins than other cell types for a few reasons. First, because neurons do not divide, mutant polyQ proteins more readily accumulate in neuronal cells than in dividing non-neuronal cells. Clearance of misfolded and mutant proteins is largely carried out by the UPS and autophagy, whose activities may preferentially decrease in neurons during the course of the disease. The preferential accumulation of mutant polyQ proteins can contribute to the selective neurodegeration in polyQ diseases. This hypothesis still needs to be validated by more experimentation. Glia, which support normal neuron function, are also affected by mutant htt, and their dysfunction could lead to neuronal excitotoxicity.

Although mutant proteins in peripheral tissues can have a deleterious impact at both cellular and organ levels, the contribution of peripheral mutant proteins to the neuropathology in polyQ diseases remains to be assessed quantitatively. Most of the previous studies show correlative evidence for the pathological changes seen in the peripheral tissues of HD patients. It remains to be investigated whether these changes are primarily caused by the expression of mutant proteins in peripheral tissues or are secondary effects due to CNS damage. Transgenic mouse models have also provided compelling evidence for the deleterious effects of mutant proteins in non-neuronal cells. However, these transgenic mice often overexpress mutant proteins, a condition that does not occur in HD patients. Thus, selective expression of mutant polyQ proteins in certain types of non-neuronal cells in transgenic animals at or below the endogenous level would allow for rigorous testing of the role of mutant proteins in peripheral tissues. While additional mechanistic data are needed to show how peripheral mutant polyQ proteins contribute to disease pathology, it is likely that improving peripheral tissue function by drugs is more feasible than improving neuronal function in the CNS. Targeting non-neuronal cells may provide an alternative approach to effectively treat or slow down polyQ diseases.

\section{Acknowledgments}

This work was supported by NIH grants NS036232, AG019206, NS041669 for X.J.L. and AG031153 for S.H.L.

Conflict of Interest: None declared.

\section{References}

1 Zoghbi HY, Orr HT. Glutamine repeats and neurodegeneration. Annu Rev Neurosci 2000; 23:217-247.

2 Li SH, Li XJ. Multiple pathways contribute to the pathogenesis of Huntington disease. Mol Neurodegeneration 2006; 1:19.

3 Martin B, Golden E, Carlson OD, et al. Exendin-4 improves glycemic control, ameliorates brain and pancreatic pathologies, and extends survival in a mouse model of Huntington's disease. Diabetes 2009; 58:318-328.

4 van der Burg JM, Björkqvist M, Brundin P. Beyond the brain: widespread pathology in Huntington's disease. Lancet Neurol 2009; 8:765-774.

5 The Huntington's Disease Collaborative Research Group. A novel gene containing a trinucleotide repeat that is expanded and unstable on Huntington's disease chromosomes. Cell 1993; 72:971-983.

6 Kremer B, Goldberg P, Andrew SE, et al. World wide distribution of the Huntington disease (HD) mutation: The sensitivity and specificity of CAG repeat length assessment. New Engl J Med 1994; 330:1401-1406.

7 Vonsattel JP, Myers RH, Stevens TJ, Ferrante RJ, Bird ED, Richardson EP. Neuropathological classification of Huntington's disease. J Neuropathol Exp Neurol 1985; 44:559-577.

8 Dragatsis I, Levine MS, Zeitlin S. Inactivation of Hdh in the brain and testis results in progressive neurodegeneration and sterility in mice. Nat Genet 2000; 26:300-306.

9 Harjes P, Wanker E. The hunt for huntingtin function: interaction partners tell many different stories. Trends Biochem Sci 2003; 28:425-433.

$10 \mathrm{Li} \mathrm{SH}, \mathrm{Li}$ XJ. Huntingtin-protein interactions and the pathogenesis of Huntington's disease. Trends Genet 2004; 20:146-154.

11 Kim YJ, Yi Y, Sapp E, et al. Caspase 3-cleaved N-terminal fragments of wild-type and mutant huntingtin are present in normal and Huntington's disease brains, associate with membranes, and undergo calpain-dependent proteolysis. Proc Natl Acad Sci USA 2001; 98:12784-12789.

12 Gafni J, Ellerby LM. Calpain activation in Huntington's disease. J Neurosci 2002; 22:4842-4849.

13 Wellington CL, Ellerby LM, Gutekunst CA, et al. Caspase cleavage of mutant huntingtin precedes neurodegeneration in Huntington's disease. J Neurosci 2002; 22:7862-7872.

14 Lunkes A, Lindenberg KS, Ben-Haïem L, et al. Proteases acting on mutant huntingtin generate cleaved products that differentially build up cytoplasmic and nuclear inclusions. Mol Cell 2002; 10:259-269.

15 Qin ZH, Gu ZL. Huntingtin processing in pathogenesis of Huntington disease. Acta Pharmacol Sin 2004; 25:1243-1249.

16 DiFiglia M, Sapp E, Chase K, et al. Huntingtin is a cytoplasmic protein associated with vesicles in human and rat brain neurons. Neuron 1995; 14:1075-1081.

17 Sharp AH, Loev SJ, Schilling G, et al. Widespread expression of Huntington's disease gene (IT15) protein product. Neuron 
1995; 14:1065-1074.

18 DiFiglia M, Sapp E, Chase KO, et al. Aggregation of huntingtin in neuronal intranuclear inclusions and dystrophic neurites in brain. Science 1997; 277:1990-1993.

19 Beal MF, Ferrante R. Experimental therapeutics in transgenic mouse models of Huntington's disease. Nat Rev 2004; 5:373384.

20 Soong BW, Paulson HL. Spinocerebellar ataxias: an update. Curr Opin Neurol 2007; 20:438-446.

21 Chung MY, Ranum LP, Duvick LA, Servadio A, Zoghbi HY, Orr HT. Evidence for a mechanism predisposing to intergenerational CAG repeat instability in spinocerebellar ataxia type I. Nat Genet 1993; 5:254-258.

22 Imbert G, Saudou F, Yvert G, et al. Cloning of the gene for spinocerebellar ataxia 2 reveals a locus with high sensitivity to expanded CAG/glutamine repeats. Nat Genet 1996; 14:285-291.

23 Warrick JM, Morabito LM, Bilen J, et al. Ataxin-3 suppresses polyglutamine neurodegeneration in Drosophila by a ubiquitin-associated mechanism. Mol Cell 2005; 18:37-48.

24 Kordasiewicz HB, Thompson RM, Clark HB, Gomez CM. C-termini of P/Q-type $\mathrm{Ca} 21$ channel a1 A subunits translocate to nuclei and promote polyglutamine-mediated toxicity. Hum Mol Genet 2006; 15:1587-1599.

25 Stevanin G, Giunti P, Belal GD, et al. De novo expansion of intermediate alleles in spinocerebellar ataxia 7. Hum Mol Genet 1998; 7:1809-1813.

26 La Spada AR, Fu YH, Sopher BL, et al. Polyglutamineexpanded ataxin-7 antagonizes CRX function and induces cone-rod dystrophy in a mouse model of SCA7. Neuron 2001; 31:913-927.

27 Friedman MJ, Shah AG, Fang ZH, et al. Polyglutamine domain modulates the TBP-TFIIB interaction: implications for its normal function and neurodegeneration. Nat Neurosci 2007; 10:1519-1528.

28 Sobue G, Hashizume Y, Mukai E, Hirayama M, Mitsuma T, Takahashi A. X-linked recessive bulbospinal neuronopathy. A clinicopathological study. Brain 1989; 112:209-232.

29 Suzuki K, Katsuno M, Banno H, et al. CAG repeat size correlates to electrophysiological motor and sensory phenotypes in SBMA. Brain 2008; 131:229-239.

30 Tanaka F, Reeves MF, Ito Y, et al. Tissue-specific somatic mosaicism in spinal and bulbar muscular atrophy is dependent on CAG-repeat length and androgen receptor-gene expression level. Am J Hum Genet 1999; 65:966-973.

31 Soukup GR, Sperfeld AD, Uttner I, et al. Frontotemporal cognitive function in X-linked spinal and bulbar muscular atrophy (SBMA): a controlled neuropsychological study of 20 patients. J Neurol 2009; 256:1869-1875.

32 Sperfeld AD, Karitzky J, Brummer D, et al. X-linked bulbospinal neuronopathy. Arch Neurol 2002; 59:1921-1926.

33 Yamada M, Shimohata M, Sato T, Tsuji S, Takahashi H. Polyglutamine disease: recent advances in the neuropathology of dentatorubralpallidoluysian atrophy. Neuropathology 2006; 26:346-351.

$34 \mathrm{Yu}$ J, Ying M, Zhuang Y, et al. C-terminal deletion of the atrophin-1 protein results in growth retardation but not neurodegeneration in mice. Dev Dyn 2009; 238:2471-2478.

35 Schilling G, Wood JD, Duan K, et al. Nuclear accumulation of truncated atrophin-1 fragments in a transgenic mouse model of
DRPLA. Neuron 1999; 24:275-286.

36 Lobsiger C, Cleveland D. Glial cells as intrinsic components of non-cell-autonomous neurodegenerative disease. Nat Neurosci 2007; 10:1355-1360.

37 Shin JY, Fang ZH, Yu ZX, Wang CE, Li SH, Li XJ. Expression of mutant huntingtin in glial cells contributes to neuronal excitotoxicity. J Cell Biol 2005; 171:1001-1012.

38 Chou SY, Weng JY, Lai HL, et al. Expanded-polyglutamine Huntingtin protein suppresses the secretion and production of a chemokine (CCL5/RANTES) by astrocytes. J Neurosci 2008; 28:3277-3290.

39 Singhrao SK, Thomas P, Wood DJ, et al. Huntingtin protein colocalizes with lesions of neurodegenerative diseases: an investigation in Huntington's, Alzheimer's, and Pick's diseases. Exp Neurol 1998; 150:213-222.

40 Rosas HD, Lee SY, Bender A, et al. Altered white matter microstructure in the corpus callosum in Huntington's disease: implications for cortical "disconnection". Neuroimage 2009; 49:2995-3004.

41 Wang CE, Tydlacka S, Orr AL, et al. Accumulation of Nterminal mutant huntingtin in mouse and monkey models implicated as a pathogenic mechanism in Huntington's disease. Hum Mol Genet 2008; 17:2738-2751.

42 Tydlacka S, Wang CE, Wang X, Li S, Li XJ. Differential activities of the ubiquitin-proteasome system in neurons versus glia may account for the preferential accumulation of misfolded proteins in neurons. J Neurosci 2008; 28:13285-13295.

43 Maragakis N, Rothstein J. Mechanisms of disease: astrocytes in neurodegenerative disease. Nat Clin Pract Neurol 2006; 2:679-689.

44 Lievens JC, Woodman B, Mahal A, et al. Impaired glutamate uptake in the R6 Huntington's disease transgenic mice. Neurobiol Dis 2001; 8:807-821.

45 Behrens PF, Franz P, Woodman B, Lindenberg KS, Landwehrmeyer GB. Impaired glutamate transport and glutamateglutamine cycling: downstream effects of the Huntington mutation. Brain 2002; 125:1908-1922.

46 Arzberger T, Krampfl K, Leimgruber S, Weindl A. Changes of NMDA receptor subunit (NR1, NR2B) and glutamate transporter (GLT1) mRNA expression in Huntington's disease - an in situ hybridization study. J Neuropathol Exp Neurol 1997; 56:440-454.

47 Hassel B, Tessler S, Faull RL, Emson PC. Glutamate uptake is reduced in prefrontal cortex in Huntington's disease. Neurochem Res 2008; 33:232-237.

48 Lievens JC, Rival T, Iche M, Chneiweiss H, Birman S. Expanded polyglutamine peptides disrupt EGF receptor signaling and glutamate transporter expression in Drosophila. Hum Mol Genet 2005; 14:713-724.

49 Bradford J, Shin JY, Roberts M, Wang CE, Li XJ, Li S. Expression of mutant huntingtin in mouse brain astrocytes causes age-dependent neurological symptoms. Proc Natl Acad Sci USA 2009; 106:22480-22485.

50 Custer SK, Garden GA, Gill N, et al. Bergmann glia expression of polyglutamine-expanded ataxin-7 produces neurodegeneration by impairing glutamate transport. Nat Neurosci 2006; 10:1302-1311.

51 Ridet JL, Malhotra SK, Privat A, Gage FH. Reactive astrocytes: cellular and molecular cues to biological function. TINS 
1997; 20:570-577.

52 Brenner M, Messing A. GFAP transgenic mice. Methods 1996; 10:351-364.

53 Yamanaka K, Chun SJ, Boillee S, et al. Astrocytes as determinants of disease progression in inherited amyotrophic lateral sclerosis. Nat Neurosci 2008; 11: 251-253.

54 Sapp E, Kegel KB, Aronin N, et al. Early and progressive accumulation of reactive microglia in the Huntington disease brain. J Neuropathol Exp Neurol 2001; 60:161-172.

$55 \mathrm{Yu}$ ZX, Li SH, Evans J, Pillarisetti A, Li H, Li XJ. Mutant huntingtin causes context-dependent neurodegeneration in mice with Huntington's disease. J Neurosci 2003; 23:2193-2202.

56 Tai YF, Pavese N, Gerhard A, et al. Imaging microglial activation in Huntington's disease. Brain Res Bull 2007; 72:148-151.

57 Kim S, de Vellis J. Microglia in health and disease. J Neurosci Res 2005; 81:302-313.

58 McGeer PL, Itagaki S, Boyes BE, McGeer EG. Reactive microglia are positive for HLA-DR in the subatantia nigra of Parkinson's and Alzheimer's disease brain. Neurology 1988; 35:1285-1291.

59 Moffitt H, McPhail GD, Woodman B, Hobbs C, Bates GP. Formation of polyglutamine inclusions in a wide range of nonCNS tissues in the HdhQ150 knock-in mouse model of Huntington's disease. PLoS One 2009; 30:e8025.

60 Lanska DJ, Lanska MJ, Lavine L, Schoenberg BS. Conditions associated with Huntington's disease at death: a case-control study. Arch Neurol 1988; 45:878-880.

61 Mihm MJ, Amann DM, Schanbacher BL, Altschuld RA, Bauer JA, Hoyt KR. Cardiac dysfunction in the R6/2 mouse model of Huntington's disease. Neurobiol Dis 2007; 25:297-308.

62 Pattison J, Robbins J. Protein misfolding and cardiac disease: establishing cause and effect. Autophagy 2008; 4:821-823.

63 Pattison JS, Sanbe A, Maloyan A, Osinska H, Klevitsky R, Robbins J. Cardiomyocyte expression of a polyglutamine preamyloid oligomer causes heart failure. Circulation 2008; 117:2743-2751.

64 Podolsky S, Leopold N, Sax D. Increased frequency of diabetes mellitus in patients with Huntington's chorea. Lancet 1972; 1:1356-1358.

65 Farrer LA. Diabetes mellitus in Huntington's disease. Clin Genet 1985; 27:62-67.

66 Podolsky S, Leopold N. Abnormal glucose tolerance and arginine tolerance tests in Huntington's disease. Gerontology 1977; 23:55-63.

67 Andreassen OA, Dedeoglu A, Stanojevic V, et al. Huntington's disease of the endrocrine pancreas: insulin deficiency and diabetes mellitus due to impaired insulin gene expression. Neurobiol Dis 2002; 11:410-424.

68 Bjorkqvist M, Fex M, Renström E, et al. The R6/2 transgenic mouse models of Huntington's disease develops diabetes due to deficient $\beta$-cell mass and exocytosis. Hum Mol Genet 2005; 14:565-574.

69 Josefsen K, Nielsen MD, Jørgensen KH, et al. Impaired glucose tolerance in the R6/1 transgenic mouse model of Huntington's disease. J Neuroendocrinol 2008; 20:165-172.

70 Andreassen OA, Dedeoglu A, Ferrante RJ, et al. Creatine increases survival and delays motor symptoms in a transgenic animal model of Huntington's disease. Neurobiol Dis 2001; 8:479-491.
71 Phan J, Hickey M, Zhang P, Chesselet MF, Reue K. Adiposed tissue dysfunction tracks disease progression in two Huntington's disease mouse models. Hum Mol Genet 2009; 18:10061016.

72 Myers RH, Sax DS, Koroshetz WJ, et al. Factors associated with slow progression in Huntington's disease. Arch Neurol 1991; 48:800-804.

73 Fain JN, Del Mar NA, Meade CA, Reiner A, Goldowitz D. Abnormalities in the functioning of adipocytes from R6/2 mice that are transgenic for the Huntington's disease mutation. Hum Mol Genet 2001; 10:145-152.

74 Li SH, Yu ZX, Li CL, et al. Lack of huntingtin-associated protein-1 causes neuronal death resembling hypothalamic degeneration in Huntington's disease. J Neurosci 2003; 23:6956-6964.

75 Henry BA, Clarke IJ. Adipose tissue hormones and the regulation of food intake. J Neuroendocrinol 2008; 20:842-849.

76 Djoussé L, Knowlton B, Cupples LA, Marder K, Shoulson I, Myers RH. Weight loss in early stage of Huntington's disease. Neurology 2002; 59:1325-1330.

77 Lee ST, Chu K, Jung KH, et al. Slowed progression in models of Huntington disease by adipose stem cell transplantation. Ann Neurol 2009; 66:671-681.

78 Sathasivam K, Hobbs C, Turmaine M, et al. Formation of polyglutamine inclusions in non-CNS tissue. Hum Mol Genet 1999; 8:813-822.

79 Ribchester RR, Thomson D, Wood NI, et al. Progressive abnormalities in skeletal muscle and neuromuscular junctions of transgenic mice expressing the Huntington's disease mutation. Eur J Neurosci 2004; 20:3092-3114.

80 Strand A, Aragaki A, Shaw D, et al. Gene expression in Huntington's disease skeletal muscle: a potential biomarker. Hum Mol Genet 2005; 14:1863-1876.

81 Carter RJ, Lione LA, Humby T, et al. Characterization of progressive motor deficits in mice transgenic for the human Huntington's disease mutation. J Neurosci 1999; 19:3248-3257.

82 Luthi-Carter R, Hanson S, Strand A, et al. Dysregulation of gene expression in the R6/2 model of polyglutamine disease: parallel changes in muscle and brain. Hum Mol Genet 2002; 11:1911-1926.

83 Weydt P, Pineda VV, Torrence AE, et al. Thermoregulatory and metabolic defects in Huntington's disease transgenic mice implicate PGC-1alpha in Huntington's disease neurodegeneration. Cell Metab 2006; 4:349-362.

84 Chaturvedi RK, Adhihetty P, Shukla S, et al. Impaired PGC1alpha function in muscle in Huntington's disease. Hum Mol Genet 2009; 18:3048-3065.

85 Masuda N, Peng Q, Li Q, et al. Tiagabine is neuroprotective in the N171-82Q and R6/2 mouse models of Huntington's disease. Neurobiol Dis 2008; 30:293-302.

86 Ferrante RJ, Andreassen OA, Jenkins BG, et al. Neuroprotective effects of creatine in a transgenic mouse model of Huntington's disease. J Neurosci 2000; 20:4389-4397.

87 Martin B, Golden E, Keselman A, et al. Therapeutic perspectives for the treatment of Huntington's disease: treating the whole body. Histol Histopathol 2008; 23:237-250.

88 Miller BR, Dorner JL, Shou M, et al. Up-regulation of GLT1 expression increases glutamate uptake and attenuates the Huntington's disease phenotype in the R6/2 mouse. Neuroscience 2008; 153:329-337. 